\title{
Circuit
}

Musiques contemporaines

\section{Les femmes et la musique : deux livres révélateurs}

\section{Cléo Palacio-Quintin}

Volume 22, numéro 3, 2012

URI : https://id.erudit.org/iderudit/1014233ar

DOI : https://doi.org/10.7202/1014233ar

Aller au sommaire du numéro

Éditeur(s)

Les Presses de l’Université de Montréal

ISSN

1183-1693 (imprimé)

1488-9692 (numérique)

Découvrir la revue

Citer ce compte rendu

Palacio-Quintin, C. (2012). Compte rendu de [Les femmes et la musique : deux livres révélateurs]. Circuit, 22(3), 83-89. https://doi.org/10.7202/1014233ar d'utilisation que vous pouvez consulter en ligne.

https://apropos.erudit.org/fr/usagers/politique-dutilisation/ 


\title{
Les femmes et la musique : deux livres révélateurs
}

\author{
Compte rendu de Cléo Palacio-Quintin
}

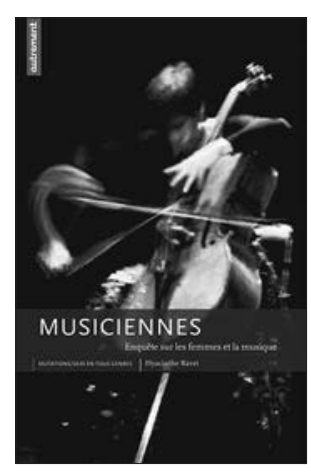

\section{Hyacinthe Ravet}

Musiciennes: enquête sur les femmes et la musique

Paris, Éditions Autrement, coll. "Mutations/Sexe en tous genres", $n^{0} 266$, 2011, 336 p.

et

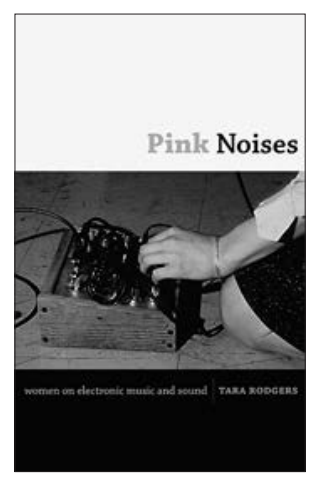

\section{Tara Rodgers}

\section{Pink Noises: Women on Electronic} Music and Sound

Durham \& London, Duke University Press, 2010, 322 p.

Malgré l'avancement des idéologies féministes depuis des décennies, la place des femmes dans le monde musical professionnel demeure encore marginale en ce début de XXI ${ }^{e}$ siècle, davantage que dans d'autres domaines artistiques. Hyacinthe Ravet et Tara Rodgers ont consacré plusieurs années de recherches afin de pouvoir témoigner de la réalité à laquelle font face de nombreuses femmes qui se consacrent à une carrière musicale dans le milieu orchestral ou celui de la musique électronique. Ces deux livres font état de l'évolution de la place des femmes en musique, mais nous font aussi réaliser à quel point l'égalité des sexes est encore une utopie dans ce domaine, malgré que les femmes soient aujourd'hui majoritaires à étudier la musique dans les écoles et conservatoires².

1. Dans le même esprit, Circuit a publié en 2009 un numéro thématique consacré aux compositrices: Composer au féminin, vol. 19, nº 1 .

2. Les étudiantes sont majoritaires dans les écoles de musique et conservatoires de France selon les statistiques récentes compilées par Hyacinthe Ravet au cours de sa recherche. II semble que cette situation soit assez généralisée en Occident. 
3. Ravet, 2011, p. 7.

4. En 2011, l'Orchestre philharmonique de Vienne comptait seulement quatre femmes titulaires sur 128 membres. Trois autres étaient en attente de titularisation. Ibid., p. 8.

5. Ibid., p. 9.

6. Ibid., p. 12

7. Ibid., p. 14.

\section{Musiciennes: enquête sur les femmes et la musique de Hyacinthe Ravet}

27 février. Anna Lelkes, la harpiste de l'Orchestre philharmonique de Vienne, est titularisée. Au pupitre depuis vingt ans, faute de candidat masculin, elle est enfin reconnue comme membre à part entière et devient la première femme à faire partie intégrante de cet orchestre. [...] ... en cette année $1997^{3}$.

À la fois sociologue, musicologue et musicienne, Hyacinthe Ravet a mené une vaste enquête pendant près de quinze ans sur l'accès des femmes à la profession de musicienne d'orchestre, en se penchant particulièrement sur la réalité des interprètes clarinettistes. Les orchestres permanents français, qui ont ouvert leurs portes aux effectifs féminins à partir des années 1970, comptent aujourd'hui un tiers de femmes. La situation est cependant beaucoup moins reluisante dans les formations germaniques autogérées qui se sont longtemps opposées à leur présence, comme en témoigne l'anecdote présentée en prologue du livre4. Encore aujourd'hui, quoique les femmes soient majoritaires parmi les chanteurs professionnels, dans le domaine instrumental, on trouve «moins de deux musiciennes professionnelles sur dix [musiciens] $]^{5}$.

Le livre de Ravet trace le portrait sociohistorique du développement de la présence des femmes dans le milieu de la musique classique. À travers neuf chapitres, elle nous dévoile l'image sociale de cette activité, en adoptant des points de vue micro- et macro- sociaux. Sa perspective est sociologique, mais s'appuie sur les connaissances musicologiques «issues des travaux d'histoire de la musique, indispensables pour éclairer l'analyse et mettant l'accent sur les significations symboliques à l'œuvre dans cet univers ${ }^{6} »$.

L'ouvrage repose donc sur la présentation des résultats d'une enquête en plusieurs volets mobilisant différents types de données: sources historiques, données de cadrage statistiques, réponses de musicien-ne-s d'orchestre professionnel-le-s à un questionnaire, entretiens et récits biographiques, données issues de l'observation?

Son survol débute par l'Antiquité, à la recherche des musiciennes «nonnes, trobaïritz et jongleresses» (chapitre 1). Elle élabore ensuite sur le «sexe des instruments» (chapitre 2), et plus loin sur les notions d'instruments «féminins » et «masculins» (chapitre 4), ce qui nous permet d'entrevoir pourquoi les femmes jouant de certains instruments ont plus rapidement rejoint les rangs des orchestres, alors que d'autres instruments demeurent des «chasses gardées» masculines.

«À l'école de la musique» (chapitre 3) relate l'historique de l'enseignement musical institutionnel en France, de la fondation du Conservatoire de 
Paris en 1795 à aujourd'hui. On y découvre que pendant la période de 1822 à 1842 (alors que l'établissement est dirigé par Cherubini), «la proportion de femmes s'élève progressivement et se stabilise à la moitié de l'effectif masculin $^{8}{ }^{»}$. On s'assure cependant que les étudiants des deux sexes se côtoient le moins possible en dehors des répétitions d'ensembles et des concerts: classes distinctes, pensionnats séparés, même l'entrée au Conservatoire se fait par différentes portes. Les femmes sont alors nombreuses à étudier le piano et le chant (pour les besoins de l'opéra évidemment), mais n'ont pas droit aux classes de composition, d'instruments à cordes, ni d'instruments à vent. Quelques-unes vont peu à peu se faufiler dans les classes de harpe et d'orgue. Les premières femmes récompensées en composition le seront vers 1860, mais ne seront admises au Prix de Rome qu'à partir de 1903. Il faudra attendre 1950 pour qu'une femme accède à la classe de direction d'orchestre.

Le chapitre 4 porte principalement sur la position de la clarinette à l'orchestre, et nous présente, après les «défricheuses» $(\sim 1970)$, les trois vagues de clarinettistes ayant fait évoluer la place des femmes dans cette profession. Ravet les qualifie de: "pionnières » ( 1980), « discrètes » $(\sim 1990)$ et "paritaires» ( 2000). Des portraits et témoignages révélateurs accompagnent cette analyse. La plupart des pionnières, premières à avoir obtenu des postes d'orchestre permanents, considèrent que l'audition derrière un paravent leur a permis de réussir. Ce sujet sera d'ailleurs développé dans le chapitre 5 consacré aux «Genre et carrières musicales», dans lequel sont évoqués les nombreux facteurs qui influencent la carrière d'une femme musicienne (modèles, discrimination, économie, maternité...).

Les chapitres 6 et 7 se penchent spécialement sur l'orchestre, en exposant la «lente ouverture» de celui-ci aux femmes, puis la question du «microcosme hiérarchisé ». La discrimination demeure le principal obstacle à l'accession des femmes à des postes de responsabilité. L'auteure résume d'ailleurs de nombreux témoignages par la formule suivante: «Nous autres femmes devons être compétentes à $200 \%$ à l'entrée; une fois engagées, on jugera notre valeur à $50 \%$ de celle des hommes moyens9. »

Finalement les deux derniers chapitres abordent la question des rôles et les attributs symboliques qui s'y rattachent. Le chapitre 9, «Diriger », présente les difficultés auxquelles les femmes font face pour accéder aux postes représentant des figures d'autorité musicales, sociales et professionnelles: chef d'orchestre, professeur, chef de pupitre, leader... «[C]omme dans d'autres sphères de pouvoir, les orchestres rechignent - souvent de manière implicite, mais parfois en le proclamant ouvertement - à accorder ce rôle aux femmes, laissant transparaître des enjeux multiples à la fois symboliques et
8. Ravet, 2011, p. 69. 
10. Ravet, 2011, p. 227

11. Ibid., p. 13.

12. Les archives du site Pinknoises com (mis en ligne en 2000 et ensuite mis à jour pendant quelques années) sont maintenant accessibles sur le lien $<$ www.safety-valve.org/pn/pn_home. html>. On y trouve plusieurs entrevues, quelques essais, une généreuse bibliographie thématique couvrant les différents aspects de "musique, genre et technologies", ainsi que de nombreux liens vers des sites de femmes musiciennes. très concrets ${ }^{10}$. D Dans l'ultime chapitre «Créer », on remet en perspective la distribution des rôles en lien avec le pouvoir créateur qui leur est associé, en passant par les «Démiurges et créateurs», «Émotions et magie » et «Pliures et "plis" du social».

L'annexe bien documentée présente de nombreuses statistiques sous forme de tableaux faciles à consulter, une méthodologie détaillée, un glossaire, une imposante bibliographie et un index. Ce livre nous trace donc un portrait très complet de la situation des femmes dans le milieu de la musique classique en général, et particulièrement dans les orchestres, en nous donnant une perspective sociohistorique globale.

Dans la perspective qui est la mienne, il s'agit davantage de saisir la spécificité des univers de la création et de l'interprétation à travers la transformation sociohistorique des représentations et des pratiques musicales, d'articuler faits matériels et phénomènes symboliques" ${ }^{11}$.

\section{Pink Noises: Women on Electronic Music and Sound de Tara Rodgers}

Dans la plupart des ouvrages concernant l'histoire de la musique électronique, pratiquement aucune femme n'est mentionnée, bien qu'il y ait toujours eu des créatrices utilisant ce média. Souhaitant remédier à cette situation, en l'an 2000, Tara Rodgers mettait en ligne le site web Pinknoises.com ${ }^{12}$, pour rassembler des entrevues réalisées auprès de nombreuses femmes œuvrant dans différentes sphères de la musique électronique: DJ, remixers, compositrices, improvisatrices, luthières, conceptrices d'installations sonores et de performances. Elle souhaitait ainsi promouvoir la musique électronique auprès des femmes et filles, en leur présentant des modèles et des ressources pour leur rendre la production musicale plus accessible.

Le livre s'inscrit en continuité avec ce projet, en présentant vingt-quatre entrevues, avec autant de femmes engagées dans des démarches artistiques utilisant différents outils électroniques. Qu'elles soient DJ, artistes sonores ou qu'elles fassent de la musique électronique à l'aide de synthétiseurs analogiques ou d'ordinateurs, Rodgers s'intéresse à leur parcours personnel, leur méthode de création, et les interroge sur la réalité féminine qui se reflète d'une façon ou d'une autre dans leur travail ou dans l'avancement de leur carrière. Certaines préfèrent ne pas discuter ou mettre l'accent sur la question du genre, considérant que cela irait à l'encontre de leur souhait de gagner une reconnaissance en tant qu' «artiste» et non en tant que «femme artiste marginalisée ». D’autres, au contraire, considèrent que leur esprit de 
revendication féministe fait partie intégrante de leur progression dans ce monde d'hommes.

Certaines traditions musicales ont un bagage culturel qui peut générer de multiples axes de discrimination, autant dans les médias que dans les contextes professionnels. Les entrevues explorent alors parfois l'intersectionnalité du genre avec d'autres aspects identitaires: race, ethnicité, classe sociale, orientation sexuelle. Pour plusieurs des femmes interviewées, ces autres aspects identitaires sont tout aussi, sinon plus importants que celui du genre. Tara Rodgers est inspirée par les différentes tendances féministes, et malgré les opinions parfois divergentes révélées dans ces entretiens, elle relie son livre aux Feminist Waves, faisant d'ailleurs le parallèle avec l'idée de sound waves (les ondes sonores étant à la fois pression et mouvement en interaction, pouvant se réverbérer à travers l'espace en influençant les trajectoires d'autres ondes).

Many artists may not identify as feminist, because of personal or cultural reasons for feeling excluded from what they understand feminism to be. [...] There may well be important differences between how the artists situate their work and politics and how I frame the project here. It is my hope that the book will demonstrate how such differences - as well as various strategies and combinations of individual achievement and collective organization - can challenge and fortify feminist movement' ${ }^{13}$.

Mais l'aspect féministe n'est pas le seul point de vue intéressant ici. Ce livre met l'accent sur la substance et la diversité du travail musical accompli par de nombreuses musiciennes créatrices aux esthétiques contrastées, toutes en lien avec l'usage de la technologie électronique et numérique. Très peu d'ouvrages répertorient l'apport des femmes en ce domaine, donc, même s'il ne faisait que ça, ce serait déjà beaucoup.

Différentes générations sont représentées parmi la variété d'artistes interviewées, ce qui nous offre une perspective très riche, qui éclaire notre vision de la pratique de la musique électronique et de la culture contemporaine. La présentation ne se fait pas de façon chronologique ni en regroupant les femmes par tendances musicales, mais Rodgers a plutôt rassemblé par quatre les différents entretiens, en six parties nommées selon des aspects esthétiques importants dans la démarche de ces artistes. Ce choix judicieux révèle ainsi au lecteur des intérêts artistiques communs parmi des femmes ayant des pratiques musicales très diversifiées. Il serait trop long de nommer ici les vingtquatre protagonistes du livre, mais j'en présente brièvement quelques-unes afin de donner une idée de la richesse de ces témoignages, et surtout des différentes perspectives qu'elle nous permet d'appréhender. 
Dans la première partie, Time and Memory, on retrouve, entre autres, deux pionnières: Pauline Oliveros (Houston, Texas, 1932), reconnue pour ses œuvres électroacoustiques depuis les années 1960, et ses nombreux écrits philosophiques, féministes et humanistes, en particulier sur le Deep listening; Éliane Radigue (Paris, France, 1932), formée aux techniques électroacoustiques auprès de Pierre Schaeffer et Pierre Henry au Studio d'essai de la Radiodiffusion-télévision française (RTF) à la fin des années 1950, sa musique, créée par des sons de synthétiseurs enregistrés sur bande, modulés et filtrés, prend la forme de sons soutenus qui évoluent très lentement. La seconde partie, Space and Perspective, nous fait découvrir Maria Chavez (Lima, Pérou, 1980), improvisatrice aux tables tournantes à l'approche bruitiste, elle produit des sons texturés à l'aide de disques vinyles et autres surfaces préparées.

La troisième partie, Nature and Synthetics, présente: Christina Kubisch (Brême, Allemagne, 1948), créatrice de sculptures sonores et installations, elle développe des techniques d'induction électromagnétique qui permettent l'interaction entre des écouteurs spéciaux et des fils électriques distribués dans l'espace de projection de ses œuvres; Jessica Rylan (Boston, États-Unis, 1974) fabrique des synthétiseurs modulaires avec des circuits analogiques qu'elle utilise dans ses performances. Le regroupement de la quatrième partie, Circulation and Movements, inclut: Giulia Loli (DJ Mutamassik) (Italie, 1973), née de mère égyptienne, elle combine des musiques traditionnelles égyptiennes et panafricaines avec des rythmes de hip-hop et des revendications politiques dans ses sessions de DJing.

Laetitia Sonami (Paris, France, 1957), pionnière dans l'usage de nouvelles interfaces gestuelles pour la création sonore, joue depuis 1991 avec son Lady's glove, un gant augmenté de capteurs qui permet d'utiliser des mouvements subtils des doigts pour contrôler en direct des sons, des dispositifs mécaniques et des éclairages. Elle se retrouve dans la catégorie de la cinquième partie, Language, Machines, Embodiement, tout comme Antye Greie (Halle-Neustadt, Allemagne de l'Est, 1969), qui se qualifie de poem producer puisqu'elle crée des textures rythmiques électroniques à partir de textes et explore ainsi le sound as language et le language as sound. Finalement, dans la sixième partie, Alone/Together, on retrouve, entre autres, Le Tigre, un groupe de musique électronique et de performance féministe, fondé en 1998, dont la musique est qualifiée d'electroclash.

Cet aperçu devrait vous convaincre de l'intérêt de lire les entretiens que Tara Rodgers a menés avec ces artistes et les quinze autres. Le livre contient également un glossaire qui sera très utile aux néophytes en musique 
électronique. On apprécie particulièrement la riche discographie compilée par Rodgers en consultant les femmes qui ont participé au livre. On y trouve une panoplie de genres et pratiques de musique électronique, DJing et art sonore, pour la plupart des musiques expérimentales et underground qu'on ne retrouvera pas chez tous les disquaires ${ }^{14}$. La bibliographie déborde largement du contexte musical et réfère à de nombreux ouvrages féministes, sociologiques et ethnologiques, et un index très détaillé permet de cibler précisément les sujets qui nous interpellent particulièrement.

Le livre Pink Noises, au titre très approprié, nous fait donc découvrir une multitude de démarches musicales originales au féminin. Ici le terme rose est un marqueur social de la différence féminine, et le bruit peut être considéré comme un facteur de perturbation au potentiel productif. Cependant, en physique et en ingénierie audio, le «bruit rose » est une variation du «bruit blanc» (signal aléatoire qui contient toutes les fréquences audibles), qui est filtré afin d'obtenir une intensité constante par octave, permettant ainsi de mettre l'accent sur les basses fréquences et le rendant plus adapté à la perception humaine. Le bruit rose est donc fréquemment utilisé, et indispensable pour tester et calibrer des équipements audio, mais bien sûr, on ne voudra ensuite pas l'entendre dans une production musicale. Tara Rodgers propose ainsi une définition du terme qui résume bien l'esprit de son livre: "pink noises: sonic interventions from multiple sources, which destabilize dominant gendered discourses and work toward equal power distributions in the cultural arenas where sounds reverberate ${ }^{15}$ ».

Les livres de Hyacinthe Ravet et Tara Rodgers permettent effectivement de redonner une place de choix aux femmes musiciennes trop souvent dans l'ombre. Ravet et Rodgers révèlent ainsi au grand jour l'importance de leurs contributions, non seulement dans les sphères musicales et artistiques, mais également sur le plan social, où l'égalité des sexes est là aussi, malheureusement, encore une utopie.
14. Tara Rodgers recommande plusieurs distributeurs et maisons de disques qui permettent de se procurer certains de ces enregistrements en ligne. Rodgers, 2010, p. 295

15. Ibid., p. 19. 